\title{
Nested row-column designs for near-factorial experiments with two treatment factors and one control treatment
}

\author{
R. A. Bailey ${ }^{\mathrm{a}}$, A. Lacka $^{\mathrm{b}}$ \\ ${ }^{a}$ School of Mathematical Sciences, Queen Mary, University of London, Mile End Road, \\ London, E1 4NS, UK (emerita) and School of Mathematics and Statistics, University of \\ St Andrews, North Haugh, St Andrews, Fife, KY16 9SS, UK \\ ${ }^{b}$ Department of Mathematical and Statistical Methods, Poznan University of Life \\ Sciences, Wojska Polskiego 28, 60-637 Poznan, Poland
}

\begin{abstract}
This paper presents some methods of designing experiments in a block design with nested rows and columns. The treatments consist of all combinations of levels of two treatment factors, with an additional control treatment.

Keywords: Block designs with nested rows and columns, Control treatment, Mixed model, Near-factorial experiments, Supplemented balance, Supplemented partial balance

2008 MSC: 62K10, 62K15
\end{abstract}

\section{Introduction}

We consider nested row-column designs, which are often used in agricultural experiments. The plots are arranged in $n_{1}$ rectangular blocks, each of which has $n_{2}$ rows and $n_{3}$ columns.

The treatments consist of complete factorial combinations of two treatment factors, plus an untreated control. This is also common in agricultural experiments. The treatment factors are $T$ and $U$, with $t$ and $u$ levels respectively. Thus the total number $v$ of treatments satisfies $v=w+1$, where $w=t u$.

Email address: rab24@st-andrews.ac.uk (R. A. Bailey)

$U R L:$ http://www-circa.mcs.st-andrews.ac.uk/ rab (R. A. Bailey) 
One natural context for these designs is plant protection experiments. Here the factors $T$ and $U$ are different types of plant protection product, such as fungicide, insecticide, plant growth regulator, rodenticide or herbicide, and their levels are different non-zero concentrations. Although it is not practical to consider using only one of the two protection products, an untreated control is needed for both scientific and regulatory reasons (FAO, 2006; Hedayat et al., 1988). Here the blocks are fields, or parts thereof, and the rows and columns are real rows and columns on the ground.

In a similar agricultural context, the levels of $T$ are different non-zero quantities of fertilizer, the levels of $U$ are different times for applying the fertilizer, and the control treatment is 'no fertilizer'. This situation happens whenever $T$ is quantitative, $U$ is qualitative, and the levels of $U$ are irrelevant when the quantity of $T$ is zero: for example, $U$ may be type of chemical, time of application, or part of the plant to be treated. See Cochran and Cox (1957, Chapters 3 and 4) and Bailey (2008, Chapter 10).

Clinical trials of medication for chronic diseases give another practical context. The blocks are health centres, the rows are patients and the columns are time periods. The levels of factor $T$ are different doses of some drug, and the levels of $U$ are different regimes of administering the drug, such as solid or liquid, time of day, one full dose or two half doses, and so on. Again, a placebo control is often required: see Matthews (2006, Chapter 1).

The paper presents several constructions of designs with our assumed structure. All these designs have general balance and control orthogonality, as described in Section 2. We also assume that there is an association scheme on the non-control treatments with $a$ associate classes. Five different schemes suitable for two treatment factors are considered in Section 2.

Thus our designs for near-factorial experiments can be classified by the association schemes on the non-control treatments.

For each of these classes, Section 3 gives some constructions of designs with certain specified parameters. Section 3.1 shows two constructions of designs with supplemented balance $(a=1)$. They are based on some properties of Latin squares and give designs with $n_{2}=n_{3}$.

Section 3.2 gives constructions of supplemented group-divisible designs, for which $a=2$. In Sections 3.3 and 3.4 various constructions for $a=3$ are shown. Section 3.3 gives two constructions of supplemented extended groupdivisible designs; again, $n_{2}=n_{3}$. For all the designs presented in Sections 3.1-3.3, all basic constrasts have, in the bottom stratum, either full efficiency or efficiency tending to one when the number of treatments increases. In Sec- 
tion 3.4 one construction of supplemented rectangular designs is considered. Section 3.5 gives two constructions which may be used when the numbers of levels of both experimental factors are the same.

Of course, for each of these constructions, the design should be randomized before being used for an experiment. The blocks should be randomized, the rows randomized independently within each block, and the columns within each block randomized independently of the rows.

Depending on the practical context, the experimenter may be most interested in comparing all active treatments with control, may value all treatment contrasts equally, may be more interested in the main effects of $T$ and $U$ than in their interaction, or may be more interested in the interaction than in the main effects. For each construction, we show how to calculate the variance of these estimators, so that the experimenter can choose among different designs according to the priorities of the experiment.

Section 4 compares the proposed constructions with other constructions in the literature and discusses the choice of an adequate design for the experiment. Examples presented here compare designs with the same parameters. These show that no single design is always best: it depends on the purpose of the experiment.

\section{General properties of the designs}

Denote the control treatment by 0 and the others by $1, \ldots, w$, which will sometimes be replaced by appropriate factorial combinations. Put $n=$ $n_{1} n_{2} n_{3}$, so that $n$ is the total number of plots.

\subsection{Information matrices}

Data from an experiment using a nested row-column design is usually analysed by using a mixed model (see Bailey and Williams, 2007; Łacka et al., 2009). However, the blocks, rows and columns give what Nelder (1965a) called an orthogonal block structure, which is defined by the block factor $B$ with $n_{1}$ levels, the row factor $R$ with $n_{2}$ levels for each level of $B$, and the column factor $C$ with $n_{3}$ levels for each level of $B$. These define four strata apart from the grand mean. These are the between-blocks stratum, which we shall also denote by $B$ or 1 ; the between-rows-within-blocks stratum $R[B]$ or 2 ; the between-columns-within-blocks stratum $C[B]$ or 3 , and the so-called bottom stratum or rows-by-columns stratum $R \# C[B]$ or 4 . All our designs have general balance, in the sense of Houtman and Speed (1983); Mejza 
(1992); Nelder (1965b, 1968), so the data may be analysed by the methods proposed by Mejza and Mejza (1994); Nelder (1968).

Let $\mathbf{N}_{1}$ be the $v \times n_{1}$ incidence matrix for treatments in blocks: the entries show how often each treatment occurs in each block. Let $\mathbf{N}_{2}, \mathbf{N}_{3}$ and $\mathbf{N}_{4}$ be the incidence matrices for rows, columns and plots respectively, so that $\mathbf{N}_{4}$ defines the design completely and $\mathbf{N}_{4} \mathbf{N}_{4}^{\prime}$ is equal to the diagonal matrix $\mathbf{R}$ of treatment replications. Denote by $\mathbf{R}^{-1 / 2}$ the diagonal matrix whose non-zero entries are the inverses of the positive square roots of the treatment replications. General balance means that the matrices $\mathbf{R}^{-1 / 2} \mathbf{N}_{k} \mathbf{N}_{k}^{\prime} \mathbf{R}^{-1 / 2}$, for $k=1, \ldots, 4$, all commute with each other.

Let $\mathbf{I}_{v}$ be the identity matrix of order $v$, let $\mathbf{J}_{v}$ be the $v \times v$ matrix whose entries are all equal to 1 , and let $\mathbf{r}$ be the column vector of treatment replications. The information matrices $\mathbf{C}_{k}$ in strata $1-4$ are given by: $\mathbf{C}_{1}=$ $\left(n_{2} n_{3}\right)^{-1} \mathbf{N}_{1} \mathbf{N}_{1}^{\prime}-n^{-1} \mathbf{r}^{\prime} \mathbf{r} ; \mathbf{C}_{2}=n_{3}^{-1} \mathbf{N}_{2} \mathbf{N}_{2}^{\prime}-\left(n_{2} n_{3}\right)^{-1} \mathbf{N}_{1} \mathbf{N}_{1}^{\prime} ; \mathbf{C}_{3}=n_{2}^{-1} \mathbf{N}_{3} \mathbf{N}_{3}^{\prime}-$ $\left(n_{2} n_{3}\right)^{-1} \mathbf{N}_{1} \mathbf{N}_{1}^{\prime}$; and $\mathbf{C}_{4}=\mathbf{R}-n_{2}^{-1} \mathbf{N}_{3} \mathbf{N}_{3}^{\prime}-n_{3}^{-1} \mathbf{N}_{2} \mathbf{N}_{2}^{\prime}+\left(n_{2} n_{3}\right)^{-1} \mathbf{N}_{1} \mathbf{N}_{1}^{\prime}$. General balance implies that the matrices $\mathbf{R}^{-1 / 2} \mathbf{C}_{k} \mathbf{R}^{-1 / 2}$, for $k=1, \ldots, 4$, all commute with each other. Thus they have common eigenvectors. For any such vector, its eigenvalue for $\mathbf{R}^{-1 / 2} \mathbf{C}_{k} \mathbf{R}^{-1 / 2}$ is called its canonical efficiency factor in stratum $k$ : see also Ceranka and Mejza (1979); Pearce et al. (1974). The vector $\mathbf{1}_{v}$ is always such an eigenvector; we are usually interested in the other eigenvectors, which all correspond to treatment contrasts. For each eigenvector, the canonical efficiency factors are non-negative and sum to 1, so they are also called the proportions of information, for the corresponding contrast, in each stratum.

In this paper, we are particularly interested in estimation using the bottom stratum, so from now on we write $\mathbf{C}_{4}$ simply as $\mathbf{C}$.

\subsection{Control orthogonality and supplemented partial balance}

All of our designs have two special properties, which we now define. There are positive integers $m_{2}$ and $m_{3}$ such that the control treatment occurs $m_{2}$ times in each row and $m_{3}$ times in each column. Hence $n_{2} m_{2}=n_{3} m_{3}$, and the overall replication $r_{0}$ of the control satisfies $r_{0}=n_{1} n_{2} m_{2}$. This condition ensures that the contrast between the control and the other treatments is orthogonal to both rows and columns, so it has full efficiency in the bottom stratum. We call this condition control orthogonality.

For the second property, we assume that there is an association scheme $\mathcal{S}$ on the $w$ non-control treatments: see Bailey (2004). For $k=1,2$ and 3 separately, the off-diagonal entries in row 0 of $\mathbf{N}_{k} \mathbf{N}_{k}^{\prime}$ are all equal, and, if 
$1 \leq i \leq j \leq w$, the value of the entry in row $i$ and column $j$ of $\mathbf{N}_{k} \mathbf{N}_{k}^{\prime}$ depends only on the associate class of $\mathcal{S}$ containing the pair $(i, j)$. The condition is phrased in this way to allow the possibility that rows, columns or blocks have repeated non-control treatments, although our constructions in Section 3 do not give such repeats in either rows or columns. Furthermore, each noncontrol treatment has replication $r$, where $w r+r_{0}=n$ (this does not follow from the other conditions if blocks are not binary). If $\mathcal{S}$ has a single associate class then the design has supplemented balance in the sense of Pearce (1960). Otherwise, we say that the design has supplemented partial balance, as this is a special case of the supplemented partial balance introduced by Bailey (2004, Section 12.3).

Under these conditions, the information matrix $\mathbf{C}$ for the bottom stratum is given by

$$
\mathbf{C}=\left[\begin{array}{cc}
w d & -d \mathbf{1}_{w}^{\prime} \\
-d \mathbf{1}_{w} & \mathbf{L}+\frac{d}{w} \mathbf{J}_{w}
\end{array}\right],
$$

where $d=r_{0} r / n$ and $\mathbf{L}$ is in the Bose-Mesner algebra $\mathcal{A}$ of $\mathcal{S}$ with $\mathbf{L} \mathbf{1}_{w}=\mathbf{0}_{w}$. Hence the Moore-Penrose generalized inverse $\mathbf{C}^{-}$of $\mathbf{C}$ is given by

$$
\mathbf{C}^{-}=\left[\begin{array}{cc}
w e & -e \mathbf{1}_{w}^{\prime} \\
-e \mathbf{1}_{w} & \mathbf{L}^{-}+\frac{e}{w} \mathbf{J}_{w}
\end{array}\right],
$$

where $d e v^{2}=1$ and $\mathbf{L}^{-}$is the Moore-Penrose generalized inverse of $\mathbf{L}$, which is also in $\mathcal{A}$.

\subsection{Variance and efficiency}

Let $\sigma^{2}$ be the stratum variance for the bottom stratum, and let $\boldsymbol{\tau}$ be the vector of $v$ treatment parameters. If $\mathbf{x}^{\prime} \boldsymbol{\tau}$ is a treatment contrast, then the variance of the estimator of $\mathbf{x}^{\prime} \boldsymbol{\tau}$, using only information in the bottom stratum, is $\mathbf{x}^{\prime} \mathbf{C}^{-} \mathbf{x} \sigma^{2}$. To compare designs, we use the variances of normalized contrasts, which have the form $\mathbf{x}^{\prime} \mathbf{C}^{-} \mathbf{x} \sigma^{2} / \mathbf{x}^{\prime} \mathbf{x}$. If there are no blocks, rows or columns then a design with the same replications gives an estimator of $\mathbf{x}^{\prime} \boldsymbol{\tau}$ with variance $\mathbf{x}^{\prime} \mathbf{R}^{-1} \mathbf{x} \sigma^{2}$. The ratio $\mathbf{x}^{\prime} \mathbf{R}^{-1} \mathbf{x} / \mathbf{x}^{\prime} \mathbf{C}^{-} \mathbf{x}$ is called the efficiency factor for this contrast in the bottom stratum: it is the same as its canonical efficiency factor when $\mathbf{x}$ is an eigenvector of $\mathbf{C}$. If this is equal to 1 , the contrast is said to have full efficiency in the bottom stratum. However, because we allow different values of $r_{0}$ and $r$, full efficiency in the bottom stratum is not the same thing as lowest variance in the bottom stratum, as 
was pointed out by Wang and Morgan (2011). We discuss this further in Section 4.

If $\mathbf{x}=\left(1,-w^{-1}, \ldots,-w^{-1}\right)^{\prime}$, which is the contrast for comparing the control treatment with the rest, then

$$
\mathbf{x}^{\prime} \mathbf{C}^{-} \mathbf{x}=w e+2 e+\frac{e}{w}=\frac{e v^{2}}{w}=\frac{n}{r_{0} r w}=\frac{1}{r_{0}}+\frac{1}{r w} .
$$

Since $\mathbf{x}^{\prime} \mathbf{x}=1+w^{-1}=v / w$, the value for the corresponding normalized contrast is $n / r_{0} r v$. These values are the same as those for a completely randomized design of the same size and replications, so this contrast has full efficiency in the bottom stratum.

Since $\mathbf{L}^{-} \in \mathcal{A}$, all diagonal entries of $\mathbf{L}^{-}$are equal. Denote this common value by $\ell$. If $\mathbf{x}$ is the contrast for comparing the control treatment with any one other treatment $i$, then

$$
\begin{aligned}
\mathbf{x}^{\prime} \mathbf{C}^{-} \mathbf{x} & =\mathbf{C}^{-}(0,0)+\mathbf{C}^{-}(i, i)-\mathbf{C}^{-}(0, i)-\mathbf{C}^{-}(i, 0) \\
& =w e+\ell+\frac{e}{w}+2 e=\frac{1}{r_{0}}+\frac{1}{r w}+\ell
\end{aligned}
$$

For the corresponding normalized contrast, divide this value by 2 .

The sum of the variances of any $v-1$ orthonormal contrasts is equal to $\operatorname{Trace}\left(\mathbf{C}^{-}\right) \sigma^{2}$ (Shah and Sinha, 1989), which is $(w e+w \ell+e) \sigma^{2}$, or

$$
\frac{w}{v}\left(\frac{1}{r_{0}}+\frac{1}{r w}+v \ell\right) \sigma^{2}
$$

A design which minimizes this value (in a given class of designs, for fixed $\sigma^{2}$ ) is called A-optimal.

Thus, for any given value of $r_{0}$, whether we seek to minimize the common variance for contrasts comparing the control treatment with any non-control treatment, or whether we seek to minimize the sum of the variances of any $v-1$ orthonormal contrasts, we should minimize $\ell$. However, when $r_{0}$ is allowed to vary, the designs which minimize one criterion may not minimize the other.

If $\mathbf{x}$ is a contrast among non-control treatments, then $\mathbf{x}=\left(0, \mathbf{y}^{\prime}\right)^{\prime}$ for some contrast $\mathbf{y}$ of length $w$. Then $\mathbf{x}^{\prime} \mathbf{C}^{-} \mathbf{x}=\mathbf{y}^{\prime} \mathbf{L}^{-} \mathbf{y}$. If the association scheme $\mathcal{S}$ has $a$ associate classes then $\mathcal{A}$ has a basis of $a+1$ primitive idempotents $\mathbf{S}_{0}, \mathbf{S}_{1}, \ldots, \mathbf{S}_{a}$, where $\mathbf{S}_{0}=w^{-1} \mathbf{J}_{w}, \sum_{i=0}^{a} \mathbf{S}_{i}=\mathbf{I}_{w}$ and $\mathbf{S}_{i} \mathbf{S}_{j}=\mathbf{0}$ if $i \neq j$. 
For a connected design, there are positive constants $\lambda_{1}, \ldots, \lambda_{a}$ such that $\mathbf{L}=r \sum_{i=1}^{a} \lambda_{i} \mathbf{S}_{i}$. Therefore, $\mathbf{L}^{-}=r^{-1} \sum_{i=1}^{a} \lambda_{i}^{-1} \mathbf{S}_{i}$. If contrast $\mathbf{y}$ is in the subspace corresponding to $\mathbf{S}_{i}$ then $\mathbf{y}^{\prime} \mathbf{L}^{-} \mathbf{y}=r^{-1} \lambda_{i}^{-1} \mathbf{y}^{\prime} \mathbf{S}_{i} \mathbf{y}=r^{-1} \lambda_{i}^{-1} \mathbf{y}^{\prime} \mathbf{y}$. In a completely randomized design with the same size, replications and error variance, the variance of the estimator of $\left(0, \mathbf{y}^{\prime}\right) \boldsymbol{\tau}$ is $r^{-1} \mathbf{y}^{\prime} \mathbf{y} \sigma^{2}$, so the efficiency factor for this contrast in the bottom stratum is $\lambda_{i}$.

The trace of an idempotent is equal to its rank, so

$$
\ell=w^{-1} \operatorname{Trace}\left(\mathbf{L}^{-}\right)=w^{-1} r^{-1} \sum_{i=1}^{a} \lambda_{i}^{-1} \operatorname{rank}\left(\mathbf{S}_{i}\right)=w^{-1} r^{-1}(w-1) A^{-1},
$$

where $A$ is the harmonic mean of the canonical efficiency factors $\lambda_{i}$, weighted according to multiplicity.

\subsection{Association schemes suitable for two treatment factors}

In our designs, the $w$ non-control treatments consist of all tu combinations of levels of a treatment factor $T$ with $t$ levels and another treatment factor $U$ with $u$ levels. For an experiment with no additional control treatment, Yates (1935) defined an equireplicate factorial design with two treatment factors $T$ and $U$ to be factorially balanced if there are constants $\lambda_{T}, \lambda_{U}$ and $\lambda_{T U}$ such that all contrasts for the main effect of $T$ have efficiency factor $\lambda_{T}$, all contrasts for the main effect of $U$ have efficiency factor $\lambda_{U}$, and all contrasts for the interaction have efficiency factor $\lambda_{T U}$. This is equivalent to saying that the design is partially balanced with respect to the rectangular association scheme $\mathrm{R}(t, u)$.

This association scheme has others as special cases. When the three efficiency factors are all equal then the design is balanced in the usual sense, which means that it is partially balanced with respect to the trivial association scheme. If only $\lambda_{U}$ and $\lambda_{T U}$ are equal then the design is partially balanced with respect to the group divisible association scheme $\operatorname{GD}(t, u)$. If $t=u$ then it is possible to have $\lambda_{T}=\lambda_{U}$ but different from $\lambda_{T U}$ : then the design is partially balanced with respect to one of the association schemes of Latin-square type.

These, and some related schemes, are used in our constructions. The rest of this section explains them in more detail.

\subsubsection{The trivial association scheme}

This has a single associate class, so that $\mathbf{L}$ and $\mathbf{L}^{-}$are both multiples of $\mathbf{I}_{w}-w^{-1} \mathbf{J}_{w}$. In this case the design has supplemented balance as well as 
control orthogonality. If $\mathbf{L}=\alpha\left(\mathbf{I}_{w}-w^{-1} \mathbf{J}_{w}\right)$ then $\mathbf{L}^{-}=\alpha^{-1}\left(\mathbf{I}_{w}-w^{-1} \mathbf{J}_{w}\right)$ and so $\boldsymbol{\ell}=(w-1) / \alpha w$. If $\mathbf{y}$ is a contrast of length $w$ then $\mathbf{y}^{\prime} \mathbf{L}^{-} \mathbf{y}=\alpha^{-1} \mathbf{y}^{\prime} \mathbf{y}$ and the variance of the corresponding normalized contrast is $\alpha^{-1} \sigma^{2}$. The efficiency factor for $\mathbf{y}$ is $\alpha / r$.

\subsubsection{The group-divisible association scheme $\operatorname{GD}(t, u)$}

This has two associate classes. Distinct treatments are first associates if they have the same level of $T$; otherwise, they are second associates. Thus there are constants $\alpha$ and $\beta$ such that

$$
\mathbf{L}=[(u-1) \alpha+(t-1) u \beta] \mathbf{I}_{w}-\alpha \mathbf{I}_{t} \otimes \mathbf{K}_{u}-\beta \mathbf{K}_{t} \otimes \mathbf{J}_{u},
$$

where $\mathbf{K}_{u}=\mathbf{J}_{u}-\mathbf{I}_{u}$. In spectral form

$$
\mathbf{L}=w \beta \mathbf{S}_{T}+u[\alpha+(t-1) \beta] \mathbf{S}_{U[T]},
$$

where $\mathbf{S}_{T}=u^{-1} \mathbf{I}_{t} \otimes \mathbf{J}_{u}-\mathbf{S}_{0}$, which is the idempotent for the main effect of $T$, and $\mathbf{S}_{U[T]}=\mathbf{I}_{w}-\mathbf{S}_{T}-\mathbf{S}_{0}$, which is the idempotent for all contrasts orthogonal to $T$. Hence

$$
\mathbf{L}^{-}=(w \beta)^{-1} \mathbf{S}_{T}+u^{-1}[\alpha+(t-1) \beta]^{-1} \mathbf{S}_{U[T]} .
$$

Therefore

$$
\ell=(t-1) w^{-2} \beta^{-1}+t(u-1)(w u)^{-1}[\alpha+(t-1) \beta]^{-1} .
$$

The normalized variance for any contrast associated with the main effect of $T$ is $(w \beta)^{-1} \sigma^{2}$, while the normalized variance for any contrast between levels of $U$ within one level of $T$ is $u^{-1}[\alpha+(t-1) \beta]^{-1} \sigma^{2}$. The corresponding efficiency factors are $\lambda_{T}=w \beta / r$ and $\lambda_{U[T]}=u[\alpha+(t-1) \beta] / r$.

\subsubsection{The extended group-divisible association scheme $\operatorname{EGD}(p, q, u)$}

This may be appropriate when $t=p q$ and a $p$-level pseudofactor $P$ groups the levels of $T$ into $p$ groups of size $q$, where $p \neq 1$ and $q \neq 1$. Now distinct treatments are first associates if they have the same level of $T$, second associates if they have the same level of $P$ but different levels of $T$, and third associates otherwise. The primitive idempotents are $\mathbf{S}_{0}, \mathbf{S}_{P}, \mathbf{S}_{T[P]}$ and $\mathbf{S}_{U[T]}$, where $\mathbf{S}_{P}=(q u)^{-1} \mathbf{I}_{p} \otimes \mathbf{J}_{q u}-\mathbf{S}_{0}, \mathbf{S}_{T[P]}=\mathbf{S}_{T}-\mathbf{S}_{P}$, and $\mathbf{S}_{T}$ and $\mathbf{S}_{U[T]}$ are as before. 
In this case there are constants $\alpha, \beta$ and $\gamma$ such that

$$
\begin{aligned}
\mathbf{L}= & {[(u-1) \alpha+(q-1) u \beta+(p-1) q u \gamma] \mathbf{I}_{w}-\alpha \mathbf{I}_{t} \otimes \mathbf{K}_{u}-\beta \mathbf{I}_{p} \otimes \mathbf{K}_{q} \otimes \mathbf{J}_{u} } \\
& -\gamma \mathbf{K}_{p} \otimes \mathbf{J}_{q u} \\
= & w \gamma \mathbf{S}_{P}+q u[\beta+(p-1) \gamma] \mathbf{S}_{T[P]}+u[\alpha+(q-1) \beta+(p-1) q \gamma] \mathbf{S}_{U[T]} .
\end{aligned}
$$

It follows that

$$
\begin{aligned}
\mathbf{L}^{-}= & (w \gamma)^{-1} \mathbf{S}_{P}+(q u)^{-1}[\beta+(p-1) \gamma]^{-1} \mathbf{S}_{T[P]} \\
& +u^{-1}[\alpha+(q-1) \beta+(p-1) q \gamma]^{-1} \mathbf{S}_{U[T]}
\end{aligned}
$$

Hence the normalized variance for contrasts between levels of $P$ is $(w \gamma)^{-1} \sigma^{2}$; for contrasts between levels of $T$ within a level of $P$ is $(q u)^{-1}[\beta+(p-1) \gamma]^{-1} \sigma^{2}$; while for contrasts between levels of $U$ within any single level of $T$ it is $u^{-1}[\alpha+(q-1) \beta+(p-1) q \gamma]^{-1} \sigma^{2}$. The corresponding efficiency factors are $\lambda_{P}=w \gamma / r, \lambda_{T[P]}=q u[\beta+(p-1) \gamma] / r$ and $\lambda_{U[T]}=u[\alpha+(q-1) \beta+(p-1) q \gamma] / r$.

Moreover,

$$
\begin{aligned}
\ell= & (p-1) w^{-2} \gamma^{-1}+p(q-1)(w q u)^{-1}[\beta+(p-1) \gamma]^{-1} \\
& +t(u-1)(w u)^{-1}[\alpha+(q-1) \beta+(p-1) q \gamma]^{-1}
\end{aligned}
$$

If $p=1$ or $q=1$ the foregoing algebra is still valid but the association scheme is $\operatorname{GD}(t, u)$. If $p=1$ then $\mathbf{S}_{P}=\mathbf{0}$, while if $q=1$ then $\mathbf{S}_{T[P]}=0$.

\subsubsection{The rectangular association scheme $\mathrm{R}(t, u)$}

This has three associate classes. Distinct treatments are first associates if they have the same level of $T$, second associates if they have the same level of $U$, and third associates otherwise. The primitive idempotents of $\mathcal{A}$ are $\mathbf{S}_{0}, \mathbf{S}_{T}, \mathbf{S}_{U}$ and $\mathbf{S}_{T U}$, where $\mathbf{S}_{T}$ is as before, $\mathbf{S}_{U}=t^{-1} \mathbf{J}_{t} \otimes \mathbf{I}_{u}-\mathbf{S}_{0}$, which corresponds to the main effect of $U$, and $\mathbf{S}_{T U}=\mathbf{I}_{w}-\mathbf{S}_{T}-\mathbf{S}_{U}-\mathbf{S}_{0}$, which corresponds to the interaction between $T$ and $U$.

Now there are constants $\alpha, \beta$ and $\gamma$ such that

$$
\begin{aligned}
\mathbf{L}= & {[(u-1) \alpha+(t-1) \beta+(t-1)(u-1) \gamma] \mathbf{I}_{w}-\alpha \mathbf{I}_{t} \otimes \mathbf{K}_{u}-\beta \mathbf{K}_{t} \otimes \mathbf{I}_{u} } \\
& -\gamma \mathbf{K}_{t} \otimes \mathbf{K}_{u} \\
= & t[\beta+(u-1) \gamma] \mathbf{S}_{T}+u[\alpha+(t-1) \gamma] \mathbf{S}_{U} \\
& +[u \alpha+t \beta+(t u-t-u) \gamma] \mathbf{S}_{T U} .
\end{aligned}
$$


The efficiency factors for the main effect of $T$, the main effect of $U$, and the interaction between $T$ and $U$ are $\lambda_{T}=t[\beta+(u-1) \gamma] / r, \lambda_{U}=u[\alpha+(t-1) \gamma] / r$ and $\lambda_{T U}=[u \alpha+t \beta+(t u-t-u) \gamma] / r$ respectively.

Furthermore,

$$
\begin{aligned}
\mathbf{L}^{-}= & t^{-1}[\beta+(u-1) \gamma]^{-1} \mathbf{S}_{T}+u^{-1}[\alpha+(t-1) \gamma]^{-1} \mathbf{S}_{U} \\
& +[u \alpha+t \beta+(t u-t-u) \gamma]^{-1} \mathbf{S}_{T U}
\end{aligned}
$$

Therefore

$$
\begin{aligned}
\ell= & (t-1)(w t)^{-1}[\beta+(u-1) \gamma]^{-1}+(u-1)(w u)^{-1}[\alpha+(t-1) \gamma]^{-1} \\
& +(t-1)(u-1) w^{-1}[u \alpha+t \beta+(t u-t-u) \gamma]^{-1}
\end{aligned}
$$

The normalized variance for any contrast associated with the main effect of $T$ is $t^{-1}[\beta+(u-1) \gamma]^{-1} \sigma^{2}$; for the main effect of $U$ it is $u^{-1}[\alpha+(t-1) \gamma]^{-1} \sigma^{2}$; and for the interaction between $T$ and $U$ it is $[u \alpha+t \beta+(t u-t-u) \gamma]^{-1} \sigma^{2}$.

\subsubsection{An association scheme defined by mutually orthogonal Latin squares}

This may be appropriate when $t=u$. Suppose that there are $h-2$ mutually orthogonal Latin squares of order $t$, where $2 \leq h \leq t+1$. Use the levels of $T$ and $U$ to the label the rows and columns, respectively, of a $t \times t$ array. Define pseudofactors $F_{1}, \ldots, F_{h}$, each with $t$ levels, as follows: $F_{1}=T$ and $F_{2}=U$; for $i=1, \ldots, h-2$, the levels of $F_{i+2}$ are the letters of the $i$-th Latin square. The pseudofactors $F_{1}, \ldots, F_{h}$ form the constraints of an orthogonal array of strength 2 .

Now distinct treatments are $i$-th associates if they have the same level of $F_{i}$, for $1 \leq i \leq h$. If $h<t+1$ then there is a further associate class, for pairs of treatments which differ in their levels of $F_{i}$ for $1 \leq i \leq h$.

Any two distinct factors $F_{i}$ and $F_{j}$ define a rectangular association scheme, with idempotents $\mathbf{S}_{i}$ and $\mathbf{S}_{j}$ defined like $\mathbf{S}_{T}$; that is, the entry in $t\left(\mathbf{S}_{i}+\mathbf{S}_{0}\right)$ is 1 if the corresponding treatments have the same level of $F_{i}$, and it is 0 otherwise. If $h=t+1$ then $\mathbf{S}_{0}+\sum_{i=1}^{h} \mathbf{S}_{i}=\mathbf{I}_{w}$; if $h<t+1$ then there is a further primitive idempotent $\mathbf{I}_{w}-\mathbf{S}_{0}-\sum_{i=1}^{h} \mathbf{S}_{i}$, which we shall denote $\mathbf{S}_{\infty}$.

Put $\mathbf{A}_{i}=t\left(\mathbf{S}_{i}+\mathbf{S}_{0}\right)-\mathbf{I}_{w}$ for $1 \leq i \leq h$, and $\mathbf{A}_{\infty}=\mathbf{J}_{w}-\mathbf{I}_{w}-\sum_{i=1}^{h} \mathbf{A}_{i}$ if $h<t+1$. If the design has supplemented partial balance with respect to this association scheme, there are constants $\alpha_{1}, \ldots, \alpha_{h}, \alpha_{\infty}$ such that

$$
\mathbf{L}=(t-1) \theta \mathbf{I}_{w}-\sum_{i=1}^{h} \alpha_{i} \mathbf{A}_{i}-\alpha_{\infty} \mathbf{A}_{\infty}
$$


where $\theta=\sum_{i=1}^{h} \alpha_{i}+(t+1-h) \alpha_{\infty}$. Hence

$$
\begin{aligned}
\mathbf{L} & =(t-1) \theta \mathbf{I}_{w}-\sum_{i=1}^{h} \alpha_{i} \mathbf{A}_{i}-\alpha_{\infty}\left(\mathbf{J}_{w}-\mathbf{I}_{w}-\sum_{i=1}^{h} \mathbf{A}_{i}\right) \\
& =(t-1) \theta \mathbf{I}_{w}+\alpha_{\infty} \mathbf{I}_{w}-\sum_{i=1}^{h}\left(\alpha_{i}-\alpha_{\infty}\right) \mathbf{A}_{i}-\alpha_{\infty} \mathbf{J}_{w} \\
& =(t-1) \theta \mathbf{I}_{w}+\alpha_{\infty} \mathbf{I}_{w}-\sum_{i=1}^{h}\left(\alpha_{i}-\alpha_{\infty}\right)\left[t\left(\mathbf{S}_{i}+\mathbf{S}_{0}\right)-\mathbf{I}_{w}\right]-\alpha_{\infty} t^{2} \mathbf{S}_{0} \\
& =t\left(\theta-\alpha_{\infty}\right) \mathbf{I}_{w}-t \sum_{i=1}^{h}\left(\alpha_{i}-\alpha_{\infty}\right) \mathbf{S}_{i}-t\left(\theta-\alpha_{\infty}\right) \mathbf{S}_{0} \\
& =t \sum_{i=1}^{h}\left(\theta-\alpha_{i}\right) \mathbf{S}_{i}+t\left(\theta-\alpha_{\infty}\right) \mathbf{S}_{\infty}
\end{aligned}
$$

Therefore

$$
\mathbf{L}^{-}=t^{-1} \sum_{i=1}^{h}\left(\theta-\alpha_{i}\right)^{-1} \mathbf{S}_{i}+t^{-1}\left(\theta-\alpha_{\infty}\right)^{-1} \mathbf{S}_{\infty} .
$$

Taking the trace gives

$$
\ell=t^{-3}(t-1)\left[\sum_{i=1}^{h}\left(\theta-\alpha_{i}\right)^{-1}+(t+1-h)\left(\theta-\alpha_{\infty}\right)^{-1}\right] .
$$

The variance for a normalized contrast for $\mathbf{S}_{i}$ (which is a contrast between levels of $F_{i}$ if $\left.i \neq \infty\right)$ is $t^{-1}\left(\theta-\alpha_{i}\right)^{-1} \sigma^{2}$, and the efficiency factor for $\mathbf{S}_{i}$ is $\lambda_{i}=t\left(\theta-\alpha_{i}\right) / r$.

\subsection{Canonical efficiency factors in other strata}

Control orthogonality and supplemented (partial) balance imply that, for $k=1,2$ and 3 ,

$$
\mathbf{C}_{k}=\left[\begin{array}{cc}
0 & \mathbf{0}_{w}^{\prime} \\
\mathbf{0}_{w} & \mathbf{L}_{k}
\end{array}\right]
$$

where $\mathbf{L}_{k} \in \mathcal{A}$ and $\mathbf{L}_{k} \mathbf{1}_{w}=\mathbf{0}_{w}$. Moreover,

$$
\sum_{k=1}^{3} \mathbf{L}_{k}+\mathbf{L}=r\left(\mathbf{I}-\frac{1}{w} \mathbf{J}_{w}\right)
$$


Thus

$$
\mathbf{C}_{k}^{-}=\left[\begin{array}{cc}
0 & \mathbf{0}_{w}^{\prime} \\
\mathbf{0}_{w} & \mathbf{L}_{k}^{-}
\end{array}\right] .
$$

The contrast for comparing the control treatment with the rest cannot be estimated in this stratum, so its canonical efficiency factor here is 0. Efficiency factors, and variances of normalized contrasts among the non-control treatments, when estimated in stratum $k$, are calculated just as in Section 2.4, by expressing $\mathbf{L}_{k}$ as a linear combination of the primitive idempotents of the association scheme $\mathcal{S}$.

\section{Constructions}

In this section we give several constructions for nested row-column designs with control orthogonality and either supplemented balance or supplemented partial balance. In each case we give the values of $n_{1}, n_{2}$ and $n_{3}$ and may specify conditions on $t$ and $u$. Section 2.5 shows that, for $k=1,2$ and 3 , it suffices to give $\mathbf{L}_{k}$ to identify $\mathbf{C}_{k}$. Sections 2.2 and 2.4 show that $\mathbf{C}_{4}$ is defined by the values of $d$ and as many of $\alpha, \beta$ are $\gamma$ are relevant: these are all shown in Table 11, along with $r$ and $r_{0}$.

\subsection{Designs with supplemented balance}

For these constructions, $n_{1}$ is arbitrary, and $n_{2}=n_{3}=w+c$ for some non-negative $c$.

\subsubsection{Construction 1}

Take $c>0$. In each block start with a Latin square of order $n_{2}$. Replace $c$ of the letters by the control, and match the rest to non-control treatments. When $c=\sqrt{w}$, Hedayat et al. (1988); Bailey (2008) show that this is the best possible for the given values of $n_{1}, n_{2}$ and $n_{3}$ for minimizing the average variance of the comparison of the control with another treatment. Now $r_{0}=c n_{1} n_{2}, r=n_{1} n_{2}$, and $\mathbf{L}_{1}=\mathbf{L}_{2}=\mathbf{L}_{3}=\mathbf{0}$. The canonical efficiency factors are shown in Table 1.

\subsubsection{Construction 2}

Now take $c=0$. In each block start with a Latin square of order $n_{2}$ using the $w$ non-control treatments as letters. The square should have the property that every letter occurs once on the main diagonal (this is possible, because $w \neq 2$ ). Then replace every treatment on the diagonal with the control. Now 
Table 1: Canonical efficiency factors in each stratum for the design given by Construction 1

\begin{tabular}{c|cc}
\hline type & with control & other \\
\hline $\mathrm{df}$ & 1 & $w-1$ \\
\hline$B$ & 0 & 0 \\
$R[B]$ & 0 & 0 \\
$C[B]$ & 0 & 0 \\
$R \# C[B]$ & 1 & 1
\end{tabular}

Table 2: Canonical efficiency factors in each stratum for the design given by Construction 2

\begin{tabular}{c|cc}
\hline type & with control & other \\
\hline $\mathrm{df}$ & 1 & $w-1$ \\
\hline$B$ & 0 & 0 \\
$R[B]$ & 0 & $\frac{1}{(w-1) w}$ \\
$C[B]$ & 0 & $\frac{1}{(w-1) w}$ \\
$R \# C[B]$ & 1 & $1-\frac{2}{(w-1) w}$
\end{tabular}

$r_{0}=w n_{1}, r=(w-1) n_{1}, \mathbf{L}_{1}=\mathbf{0}$, and $\mathbf{L}_{2}=\mathbf{L}_{3}=n_{1} w^{-1}\left(\mathbf{I}_{w}-w^{-1} \mathbf{J}_{w}\right)$. The canonical efficiency factors are in Table 2.

\subsection{Supplemented group-divisible designs}

\subsubsection{Construction 3}

Here $n_{1}=t$ and $n_{2}=n_{3}=w$.

The construction uses $t$ Latin squares of order $w$, for the $w$ non-control treatments. In the first square, replace those treatments with the first level of factor $T$ by the control. In block $j$, the control replaces all treatments with level $j$ of $T$. When $t=3$ and $u=2$, this method gives the design in Example 1.

In each block we lose exactly one level of factor $T$, so the contrasts for this factor are involved in the between-blocks stratum. The design in rows is the same as the design in columns, and both are proportional to the design in blocks, so $\mathbf{L}_{2}=\mathbf{L}_{3}=\mathbf{0}$. Also $\mathbf{L}_{1}=t^{-1}\left[(t-1) \mathbf{I}_{w}+(t-1) \mathbf{I}_{t} \otimes \mathbf{K}_{u}-\mathbf{K}_{t} \otimes \mathbf{J}_{u}\right]$. These lead to the canonical efficiency factors in Table 3.

In the first stratum, only contrasts for the main effect of $T$ are estimable, and the efficiency factor has the low value $1 / t(t-1)$, which tends to zero as $t$ increases. No contrasts can be estimated in the strata $R[B]$ or $C[B]$, because both information matrices are zero. In the bottom stratum, all constrasts for comparing levels of $U$ within any one level of $T$ are estimated with full efficiency, and contrasts for the main effect of $T$ have an efficiency factor which tends to 1 as $t$ increases. 
Table 3: Canonical efficiency factors in each stratum for the design given by Construction 3

\begin{tabular}{c|ccc}
\hline type & with control & $\begin{array}{c}\text { factor } T \\
T\end{array}$ & $\begin{array}{c}\text { factor } U \text { within } \\
\text { each level of } T \\
U[T]\end{array}$ \\
\hline $\mathrm{df}$ & 1 & $t-1$ & $t(u-1)$ \\
\hline$B$ & 0 & $\frac{1}{t(t-1)}$ & 0 \\
$R[B]$ & 0 & 0 & 0 \\
$C[B]$ & 0 & 0 & 0 \\
$R \# C[B]$ & 1 & $1-\frac{1}{t(t-1)}$ & 1
\end{tabular}

Example 1. Put $t=3$ and $u=2$. Denote by 21 the treatment which has level 2 of factor $T$ and level 1 of factor $U$; denote other combinations in a similar way. One choice of three $6 \times 6$ Latin squares in Construction 3 gives the design in Figure 1.

\begin{tabular}{|c|c|c|}
\hline Block 1 & Block 2 & Block 3 \\
\hline H $N$ r & 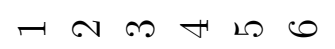 & 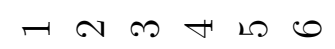 \\
\hline 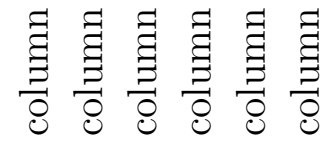 & 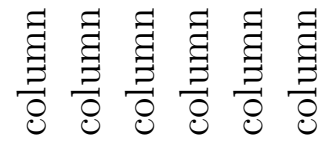 & 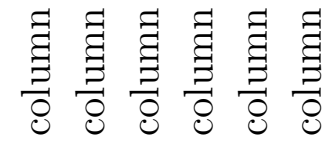 \\
\hline $\begin{array}{llllll}0 & 0 & 21 & 22 & 31 & 32\end{array}$ & $11 \quad 12 \quad 0 \quad 0 \quad 3132$ & 1112212200 \\
\hline $\begin{array}{llllll}0 & 21 & 22 & 31 & 32 & 0\end{array}$ & $\begin{array}{llllll}12 & 0 & 0 & 31 & 32 & 11\end{array}$ & $122122 \quad 0 \quad 0 \quad 11$ \\
\hline $21223132 \quad 0 \quad 0$ & $\begin{array}{llllll}0 & 0 & 31 & 32 & 11 & 12\end{array}$ & $\begin{array}{llllll}21 & 22 & 0 & 0 & 11 & 12\end{array}$ \\
\hline $223132 \quad 0 \quad 0 \quad 21$ & $\begin{array}{llllll}0 & 3132 & 11 & 12 & 0\end{array}$ & $\begin{array}{llllll}22 & 0 & 0 & 11 & 12 & 21\end{array}$ \\
\hline $\begin{array}{llllll}31 & 32 & 0 & 0 & 21 & 22\end{array}$ & $\begin{array}{llllll}31 & 32 & 11 & 12 & 0 & 0\end{array}$ & $\begin{array}{llllll}0 & 0 & 11 & 12 & 21 & 22\end{array}$ \\
\hline $\begin{array}{llllll}32 & 0 & 0 & 21 & 22 & 31\end{array}$ & $\begin{array}{llllll}32 & 11 & 12 & 0 & 0 & 31\end{array}$ & $\begin{array}{llllll}0 & 11 & 12 & 21 & 22 & 0\end{array}$ \\
\hline
\end{tabular}

Figure 1: Design in Example 1.

\subsection{Supplemented extended group-divisible designs}

The next two constructions are suitable when $t=p q$, so that $T$ may be replaced by pseudofactors $P$ and $Q$ with $p$ and $q$ levels respectively. Let $\Lambda$ be a $t \times t$ Latin square with the property that there are $q$ letters which occur on the main diagonal $p$ times each (such a square exists unless $p=1$ and $q=2$ ). For $i=1, \ldots, p$, create Latin square $\Lambda_{i}$ by allocating level $i$ of $P$ to 
the letters on the diagonal and dividing the remaining letters equally among the other levels of $P$. Within each level of $P$, levels of $Q$ can be assigned to the $q$ letters in any way. Then the main diagonal of $\Lambda_{i}$ has all the letters with level $i$ of $P$, each occurring $p$ times.

These squares serve as the basis for constructing designs with $n_{1}=p$ and $n_{2}=n_{3}=w$. For $i=1, \ldots, p$, block $i$ is constructed from Latin square $\Lambda_{i}$.

\subsubsection{Construction 4}

In block $i$, use Latin square $\Lambda_{i}$. Off the diagonal, replace letter $m$ by a $u \times u$ Latin square on the treatments which have level $m$ of $T$. On the diagonal, replace letter $m$ by a $u \times u$ square which has the treatments with level $m$ of $T$ on the diagonal and the control elsewhere. Then

$\mathbf{L}_{1}=(u-1)^{2} t^{-1} q^{-1} u^{-2}\left[(p-1) \mathbf{I}_{w}+(p-1) \mathbf{I}_{t} \otimes \mathbf{K}_{u}+(p-1) \mathbf{I}_{p} \otimes \mathbf{K}_{q} \otimes \mathbf{J}_{u}-\mathbf{K}_{p} \otimes \mathbf{J}_{u q}\right]$

and

$$
\begin{aligned}
\mathbf{L}_{2}=\mathbf{L}_{3}= & u^{-2} q^{-2}\left[(u-1)(u q-u+1) \mathbf{I}_{w}\right. \\
& \left.+(u(u-2)(q-1)-1) \mathbf{I}_{t} \otimes \mathbf{K}_{u}-(u-1)^{2} \mathbf{I}_{p} \otimes \mathbf{K}_{q} \otimes \mathbf{J}_{u}\right] .
\end{aligned}
$$

Canonical efficiency factors are shown in Table 4.

Table 4: Canonical efficiency factors in each stratum for the design given by Construction 4

\begin{tabular}{c|cccc}
\hline type & $\begin{array}{c}\text { with } \\
\text { control }\end{array}$ & $\begin{array}{c}\text { between } p \\
\text { groups of } T\end{array}$ & $\begin{array}{c}\text { between } q \\
\text { groups within } \\
\text { each } P \text { group } \\
T[P]\end{array}$ & $\begin{array}{c}\text { factor } U \\
\text { within each } \\
\text { level of } T \\
\end{array}$ \\
\hline df & 1 & $p-1$ & $p(q-1)$ & $(u-1) p q$ \\
\hline$B$ & 0 & $\frac{(u-1)^{2}}{w(w-u+1)}$ & 0 & 0 \\
$R[B]$ & 0 & 0 & $\frac{(u-1)^{2}}{w(w-u+1)}$ & $\frac{1}{w(w-u+1)}$ \\
$C[B]$ & 0 & 0 & $\frac{(u-1)^{2}}{w(w-u+1)}$ & $\frac{1}{w(w-u+1)}$ \\
$R \# C[B]$ & 1 & $1-\frac{(u-1)^{2}}{w(w-u+1)}$ & $1-\frac{2(u-1)^{2}}{w(w-u+1)}$ & $1-\frac{2}{w(w-u+1)}$
\end{tabular}


Base block 1

$\begin{array}{cccc}A_{0} & B & C & D \\ B & C_{0} & D & A \\ C & D & A_{0} & B \\ D & A & B & C_{0}\end{array}$

Base block 2

$\begin{array}{cccc}B_{0} & C & D & A \\ C & D_{0} & A & B \\ D & A & B_{0} & C \\ A & B & C & D_{0}\end{array}$

replace with:

\begin{tabular}{|c|c|c|c|c|c|c|c|c|c|c|c|c|c|c|c|}
\hline & 13 & 11 & 12 & & 23 & 21 & 22 & & 33 & 31 & 32 & & 43 & 41 & 42 \\
\hline$A-$ & 11 & 12 & 13 & $B \rightarrow$ & 21 & 22 & 23 & $C \rightarrow$ & 31 & 32 & 33 & $D \rightarrow$ & 41 & 42 & 3 \\
\hline & 12 & 13 & 11 & & 22 & 23 & 21 & & 32 & 33 & 31 & & 42 & 43 & \\
\hline & 1 & U & 0 & & & & & & & & & & 43 & 0 & \\
\hline 210 & 0 & 12 & 0 & $B_{0} \rightarrow$ & 0 & 22 & 0 & $C_{0} \rightarrow$ & 0 & 32 & 0 & $D_{0} \rightarrow$ & 0 & 42 & \\
\hline
\end{tabular}

Figure 2: Squares used in the construction in Example 2

Example 2. Take $p=q=2$ and $u=3$. Use two Latin squares of order 4, such that the diagonal of one contains letters $A$ and $C$ twice each and the diagonal in the second contains the letters $B$ and $D$ twice each. Figure 2 shows possible choices. For each letter $A-D$ build a Latin square of order 3 for the treatments with a single level of $T$. In each of the two base squares, replace all non-diagonal letters by the new Latin squares, and replace each diagonal letter by a square with the corresponding treatments on the diagonal and the control treatment elsewhere. Possible squares to use in the second stage of the construction are shown in Figure 2.

Two special cases of this construction occur when $q=1$ but $p \neq 1$ and when $p=1$ but $q \neq 1$. In both cases we lose one associate class and one group of contrasts, as the association scheme reverts to the group-divisible type. The basic contrasts are analogous to those described in the study by Lacka and Kozłowska (2009). In the first case, the design has $t$ blocks, each constructed from a Latin square of order $t$ with the same letter throughout the diagonal, different in each square: see Example 3. If $p=1$ then there is only one block, made from a single Latin square of order $t$ with all letters on the diagonal: see Example 4.

Example 3. Construction 4 for $p=4, q=1$ and $u=3$ can give the design in Figure 3, where $A, A_{0}, B, B_{0}, C, C_{0}, D$ and $D_{0}$ are given in Figure 2. 
Base block $1 \quad$ Base block $2 \quad$ Base block $3 \quad$ Base block 4

$\begin{array}{llllllllllllllll}A_{0} & B & C & D & B_{0} & A & C & D & C_{0} & B & A & D & D_{0} & B & C & A\end{array}$

$\begin{array}{llllllllllllllll}D & A_{0} & B & C & D & B_{0} & A & C & D & C_{0} & B & A & A & D_{0} & B & C\end{array}$

$\begin{array}{llllllllllllllll}C & D & A_{0} & B & C & D & B_{0} & A & A & D & C_{0} & B & C & A & D_{0} & B\end{array}$

$\begin{array}{llllllllllllllll}B & C & D & A_{0} & A & C & D & B_{0} & B & A & D & C_{0} & B & C & A & D_{0}\end{array}$

Figure 3: Design in Example 3: $A, A_{0}, B, B_{0}, C, C_{0}, D$ and $D_{0}$ are given in Figure 2

Example 4. Figure 4 shows a design made using Construction 4 for $p=1$, $q=4$ and $u=3$. Again, $A, A_{0}, B, B_{0}, C, C_{0}, D$, and $D_{0}$ are given in Figure 2.

\begin{tabular}{cccc}
\multicolumn{4}{c}{ Base block 1} \\
$A_{0}$ & $D$ & $B$ & $C$ \\
$C$ & $B_{0}$ & $D$ & $A$ \\
$D$ & $A$ & $C_{0}$ & $B$ \\
$B$ & $C$ & $A$ & $D_{0}$
\end{tabular}

Figure 4: Design in Example 4: $A, A_{0}, B, B_{0}, C, C_{0}, D$ and $D_{0}$ are given in Figure 2

\subsubsection{Construction 5}

Start like Construction 4, but make the replaced squares on the diagonal all controls. When $q=1$ this gives Construction 3 as a special case. When $p=1$ it gives another supplemented group-divisible design. For example, when $t=4$ and $u=3$ we obtain the design in Figure 4 , with $A, B, C$ and $D$ as in Figure 2 and $A_{0}, B_{0}, C_{0}$ and $D_{0}$ all being $3 \times 3$ squares of controls. Now $\mathbf{L}_{1}=p t^{-2}\left[(p-1) \mathbf{I}_{w}+(p-1) \mathbf{I}_{t} \otimes \mathbf{K}_{u}+(p-1) \mathbf{I}_{p} \otimes \mathbf{K}_{q} \otimes \mathbf{J}_{u}-\mathbf{K}_{p} \otimes \mathbf{J}_{u q}\right]$ and $\mathbf{L}_{2}=\mathbf{L}_{3}=p t^{-2}\left[(t-p) \mathbf{I}_{w}+(t-p) \mathbf{I}_{t} \otimes \mathbf{K}_{u}-p \mathbf{I}_{p} \otimes \mathbf{K}_{q} \otimes \mathbf{J}_{u}\right]$. The canonical efficiency factors are in Table 5 .

\subsection{Supplemented rectangular designs}

\subsubsection{Construction 6}

Sometimes the previous constructions require too much replication of treatments. In such a situation, if $u \neq 2$ we suggest creating a design with $n_{2}=u$ and $n_{3}=w$. All blocks have the same construction, so $n_{1}$ is arbitrary. To create each block of the design, start with Latin squares $\Lambda_{1}, \ldots, \Lambda_{t}$ of order $u$. The letters of $\Lambda_{i}$ are the treatments with level $i$ of $T$; the diagonal 
Table 5: Canonical efficiency factors in each stratum for the design given by Construction 5

\begin{tabular}{c|cccc}
\hline type & $\begin{array}{c}\text { with } \\
\text { control }\end{array}$ & $\begin{array}{c}\text { between } p \\
\text { groups of } T\end{array}$ & $\begin{array}{c}\text { between } q \\
\text { groups within } \\
\text { each } P \text { group } \\
T[P]\end{array}$ & $\begin{array}{c}\text { factor } U \\
\text { within each } \\
\text { level of } T \\
U[T]\end{array}$ \\
\hline df & 1 & $p-1$ & $p(q-1)$ & $(u-1) p q$ \\
\hline$B$ & 0 & $\frac{1}{t(t-1)}$ & 0 & 0 \\
$R[B]$ & 0 & 0 & $\frac{1}{t(t-1)}$ & 0 \\
$C[B]$ & 0 & 0 & $\frac{1}{t(t-1)}$ & 0 \\
$R \# C[B]$ & 1 & $1-\frac{1}{t(t-1)}$ & $1-\frac{2}{t(t-1)}$ & 1
\end{tabular}

elements of each square should all be different, and the levels of $U$ must be in the same positions in each square. Replace all diagonal elements with a control treatment. Put all the squares together in one row to make the block (see Example 5). Now $T$ has only one level per column, while $U$ has one level missing from each row and from each column. Hence the main effect of $T$ has some information in the columns-within-blocks stratum, and the main effect of $U$ has information in both the columns-within-blocks and rows-withinblocks strata. Every block contains all treatments. For $n_{1}$ blocks, $\mathbf{L}_{1}=\mathbf{0}$, $\mathbf{L}_{2}=n_{1} u^{-2} t^{-1}\left[(u-1) \mathbf{I}_{w}-\mathbf{I}_{t} \otimes \mathbf{K}_{u}+(u-1) \mathbf{K}_{t} \otimes \mathbf{I}_{u}-\mathbf{K}_{t} \otimes \mathbf{K}_{u}\right]$ and

$$
\begin{aligned}
\mathbf{L}_{3}= & n_{1} u^{-2} t^{-1}\left[(w-u+1)(u-1) \mathbf{I}_{w}+\left(w u-u^{2}-2 w+2 u-1\right) \mathbf{I}_{t} \otimes \mathbf{K}_{u}\right. \\
& \left.-(u-1)^{2} \mathbf{K}_{t} \otimes \mathbf{I}_{u}-(u-1)^{2} \mathbf{K}_{t} \otimes \mathbf{K}_{u}\right] .
\end{aligned}
$$

These lead to the canonical efficiency factors in Table 6 .

In this design, the main effect of $T$ has relatively low estimation efficiency in the bottom stratum. Thus, when planning the experiment, one should take $T$ to be the factor that is less interesting or, in the analysis of that group of contrasts, combine information from the third and fourth strata (Łacka et al., 2009). Other contrasts, both those for the main effect of $U$ and those for the interaction, are estimated with efficiencies which tend to 1 as the number of levels of the factor $U$ increases.

Example 5. Put $t=3$ and $u=4$ in Construction 6. One possible design is shown in Figure 5. 
Table 6: Canonical efficiency factors in each stratum for the design given by Construction 6

\begin{tabular}{c|cccc}
\hline type & with control & $\begin{array}{c}\text { factor } T \\
T\end{array}$ & $\begin{array}{c}\text { factor } U \\
U\end{array}$ & $\begin{array}{c}\text { interaction } \\
T \# U\end{array}$ \\
\hline $\mathrm{df}$ & 1 & $t-1$ & $u-1$ & $(t-1)(u-1)$ \\
\hline$B$ & 0 & 0 & 0 & 0 \\
$R[B]$ & 0 & 0 & $\frac{1}{u(u-1)}$ & 0 \\
$C[B]$ & 0 & $\frac{u-1}{u}$ & $\frac{1}{u(u-1)}$ & $\frac{1}{u(u-1)}$ \\
$R \# C[B]$ & 1 & $\frac{1}{u}$ & $1-\frac{2}{u(u-1)}$ & $1-\frac{1}{u(u-1)}$
\end{tabular}

Block 1

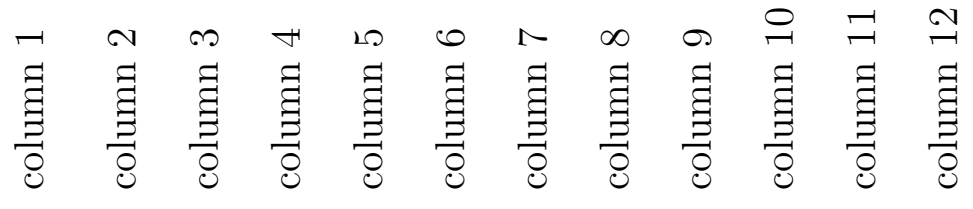

\begin{tabular}{l|cccc|cccc|cccc} 
row 1 & 0 & 11 & 13 & 12 & 0 & 21 & 23 & 22 & 0 & 31 & 33 & 32 \\
row 2 & 12 & 0 & 11 & 14 & 22 & 0 & 21 & 24 & 32 & 0 & 31 & 34 \\
row 3 & 11 & 14 & 0 & 13 & 21 & 24 & 0 & 23 & 31 & 34 & 0 & 33 \\
row 4 & 13 & 12 & 14 & 0 & 23 & 22 & 24 & 0 & 33 & 32 & 34 & 0
\end{tabular}

Figure 5: Design in Example 5 


\subsection{Designs for factors with the same number of levels}

When $t=u$, all the previous constructions may be used, and some others are available.

\subsubsection{Construction 7}

This construction requires $t$ to be a power of a prime. It gives $n_{2}=t+1$ and $n_{3}=t(t+1)$, while $n_{1}$ is arbitrary because each block is made in the same way.

For each block make $t^{2}+t+1$ columns of $t+1$ letters, so that they form a balanced incomplete block design on $t^{2}+t+1$ letters. It is often possible to find a perfect difference set of $t+1$ numbers modulo $t^{2}+t+1$. For example, $\{1,2,5,7\}$ is a perfect difference set modulo 13 because the twelve difference $1-2,1-5, \ldots, 7-5$ give every non-zero number modulo 13 exactly once. Write the perfect difference set in the first column. Obtain each successive column by adding 1 modulo $t^{2}+t+1$.

If the design has been made from a perfect difference set, every element should appear once in each row. Otherwise, make the block design from $t-1$ mutually orthogonal Latin squares of order $t$ as described in Bailey (2008, Section 11.3) and then use the technique in Bailey (2008, Section 11.10) to re-arrange the letters in each column so that each letter occurs once in each row.

Remove one column and replace all other occurrences of the letters in that column by the control. Match the remaining letters to the non-control treatments. Then $r_{0}=t(t+1) n_{1}, r=(t+1) n_{1}$ and the resulting design has supplemented balance.

Now $d=n_{1}, \mathbf{L}_{1}=\mathbf{L}_{2}=\mathbf{0}$ and $\mathbf{L}_{3}=n_{1} t(t+1)^{-1}\left(\mathbf{I}_{w}-w^{-1} \mathbf{J}_{w}\right)$, where $w=t^{2}$. Table 7 gives the canonical efficiency factors.

Table 7: Canonical efficiency factors in each stratum for the design given by Construction 7

\begin{tabular}{c|cc}
\hline type & with control & all other \\
\hline $\mathrm{df}$ & 1 & $t^{2}-1$ \\
\hline$B$ & 0 & 0 \\
$R[B]$ & 0 & 0 \\
$C[B]$ & 0 & $\frac{t}{(t+1)^{2}}$ \\
$R \# C[B]$ & 1 & $1-\frac{t}{(t+1)^{2}}$
\end{tabular}


Example 6. Take $t=u=3$, so that $t^{2}+t+1=13$. Translating the perfect difference set $\{1,2,5,7\}$ into letters in the obvious way gives the following $4 \times 13$ rectangle.

$$
\begin{array}{lllllllllllll}
A & B & C & D & E & F & G & H & I & J & K & L & M \\
B & C & D & E & F & G & H & I & J & K & L & M & A \\
E & F & G & H & I & J & K & L & M & A & B & C & D \\
G & H & I & J & K & L & M & A & B & C & D & E & F
\end{array}
$$

Removing the final column and replacing the remaining letters lexicographically, gives:

$\begin{array}{cccccccccccc}0 & 11 & 12 & 0 & 13 & 0 & 21 & 22 & 23 & 31 & 32 & 33 \\ 11 & 12 & 0 & 13 & 0 & 21 & 22 & 23 & 31 & 32 & 33 & 0 \\ 13 & 0 & 21 & 22 & 23 & 31 & 32 & 33 & 0 & 0 & 11 & 12 \\ 21 & 22 & 23 & 31 & 32 & 33 & 0 & 0 & 11 & 12 & 0 & 13\end{array}$

\subsubsection{Construction 8}

In this construction, $n_{1}$ is arbitrary, $n_{2}=t$ and $n_{3}=t^{2}$. Put the treatments into a square array, with the rows labelled by levels of $T$ and the columns labelled by levels of $U$. Use $h-2$ orthogonal Latin squares of order $t$, as described in Section 2.4.5. For the first block, let $F_{i}$ and $F_{j}$ be any two distinct pseudofactors among $F_{1}, \ldots, F_{h}$. Use Construction 6 with $F_{i}$ in place of $T$ and $F_{j}$ in place of $U$. Write the information matrices for this block with superscript (1). Then $\mathbf{L}_{1}^{(1)}=\mathbf{0}$, and the results in Table 6 give

$$
\begin{aligned}
t \mathbf{L}_{2}^{(1)} & =\mathbf{S}_{j} \\
t \mathbf{L}_{3}^{(1)} & =\mathbf{S}_{j}+(t-1)^{2} \mathbf{S}_{i}+\left(\mathbf{I}_{w}-\mathbf{S}_{0}-\mathbf{S}_{i}-\mathbf{S}_{j}\right)=t(t-2) \mathbf{S}_{i}+\mathbf{I}_{w}-\mathbf{S}_{0} \\
t \mathbf{L}^{(1)} & =\left(t^{2}-t-2\right) \mathbf{S}_{j}+(t-1) \mathbf{S}_{i}+\left(t^{2}-t-1\right)\left(\mathbf{I}_{w}-\mathbf{S}_{0}-\mathbf{S}_{i}-\mathbf{S}_{j}\right) \\
& =-\mathbf{S}_{j}-t(t-2) \mathbf{S}_{i}+\left(t^{2}-t-1\right)\left(\mathbf{I}_{w}-\mathbf{S}_{0}\right) .
\end{aligned}
$$

Subsequent blocks are made similarly, but the row and column factors may be chosen freely among $F_{1}, \ldots, F_{h}$ so long as the two pseudofactors used in each block are different. For $i=1, \ldots, h$, let $\psi(i)$ be the number of blocks in which $F_{i}$ is used in place of $T$ (bad confounding with columns), and let $\rho(i)$ be the number of blocks in which $F_{i}$ is used in place of $U$ (small confounding with rows). Treatments are orthogonal to blocks, so the matrices 
$\mathbf{L}_{1}, \mathbf{L}_{2}, \mathbf{L}_{3}$ and $\mathbf{L}$ for the whole design are obtained by summing the relevant matrices for the individual blocks. Hence $\mathbf{L}_{1}=\mathbf{0}$, and

$$
\begin{aligned}
t \mathbf{L}_{2} & =\sum_{i=1}^{h} \rho(i) \mathbf{S}_{i} ; \\
t \mathbf{L}_{3} & =t(t-2) \sum_{i=1}^{h} \psi(i) \mathbf{S}_{i}+n_{1}\left(\mathbf{I}_{w}-\mathbf{S}_{0}\right) ; \\
t \mathbf{L} & =-\sum_{i=1}^{h}[\rho(i)+t(t-2) \psi(i)] \mathbf{S}_{i}+n_{1}\left(t^{2}-t-1\right)\left(\mathbf{I}_{w}-\mathbf{S}_{0}\right) \\
& =\sum_{i=1}^{h}\left[n_{1}\left(t^{2}-t-1\right)-\rho(i)-t(t-2) \psi(i)\right] \mathbf{S}_{i}+n_{1}\left(t^{2}-t-1\right) \mathbf{S}_{\infty} .
\end{aligned}
$$

Taking generalized inverses gives

$$
\frac{n_{1}}{t} \mathbf{L}^{-}=\sum_{i=1}^{h}\left[t^{2}-t-1-\frac{\rho(i)}{n_{1}}-t(t-2) \frac{\psi(i)}{n_{1}}\right]^{-1} \mathbf{S}_{i}+\left(t^{2}-t-1\right)^{-1} \mathbf{S}_{\infty}
$$

and so

$\ell=\frac{t-1}{t n_{1}}\left[\sum_{i=1}^{h}\left[t^{2}-t-1-\frac{\rho(i)}{n_{1}}-t(t-2) \frac{\psi(i)}{n_{1}}\right]^{-1}+(t+1-h)\left(t^{2}-t-1\right)^{-1}\right]$.

To minimize this, the values $\rho(i)+t(t-2) \psi(i)$ should be chosen to be as equal as possible.

If $h=t+1=n_{1}$, we can make $\rho(i)=\psi(i)=1$ for $i=1, \ldots, t+1$, so the whole design has supplemented balance. Now $d=(t-1)(t+1) / t, \mathbf{L}_{1}=\mathbf{0}$, $\mathbf{L}_{2}=t^{-1}\left(\mathbf{I}_{w}-w^{-1} \mathbf{J}_{w}\right)$ and $\mathbf{L}_{3}=\left(t^{2}-t+1\right) \mathbf{L}_{2}$. Table 8 gives the canonical efficiency factors in this case.

Example 7. When $t=u=3$ we can use Construction 8 with $h=n_{1}=4$ to obtain a design with supplemented balance. Table 9 shows the pseudofactors $F_{1}, \ldots, F_{4}$ as an orthogonal array. Using each pseudofactor once in each role of Construction 6 gives the design in Figure 6.

If fewer blocks are used, the design still has supplemented partial balance with respect to the Latin-square type of association scheme in Section 2.4.5, 
Table 8: Canonical efficiency factors in each stratum for one design given by Construction 8

\begin{tabular}{c|cc}
\hline type & with control & all other \\
\hline $\mathrm{df}$ & 1 & $t^{2}-1$ \\
\hline$B$ & 0 & 0 \\
$R[B]$ & 0 & $\frac{1}{t\left(t^{2}-1\right)}$ \\
$C[B]$ & 0 & $\frac{t^{2}-t+1}{t\left(t^{2}-1\right)}$ \\
$R \# C[B]$ & 1 & $1-\frac{t^{2}-t+2}{t\left(t^{2}-1\right)}$
\end{tabular}

Table 9: Four pseudofactors in Example 7

\begin{tabular}{|c|c|c|c|c|c|c|c|c|c|c|c|c|}
\hline \multirow{5}{*}{$\begin{array}{l}\text { factors } \\
F_{1}=T\end{array}$} & \multicolumn{9}{|c|}{ non-control treatments } & \multirow{2}{*}{\multicolumn{3}{|c|}{ groups for each pseudofactor }} \\
\hline & 1 & 2 & 3 & 4 & 5 & 6 & 7 & 8 & 9 & & & \\
\hline & & & & & & & & & & 1 & 4 & 7 \\
\hline & 1 & 1 & 1 & 2 & 2 & 2 & 3 & 3 & 3 & 2 & 5 & 8 \\
\hline & & & & & & & & & & 3 & 6 & 9 \\
\hline \multirow{3}{*}{$F_{2}=U$} & \multirow{3}{*}{1} & \multirow{3}{*}{2} & \multirow{3}{*}{3} & \multirow{3}{*}{1} & \multirow{3}{*}{2} & \multirow{3}{*}{3} & \multirow{3}{*}{1} & \multirow{3}{*}{2} & \multirow{3}{*}{3} & 1 & 2 & 3 \\
\hline & & & & & & & & & & 4 & 5 & 6 \\
\hline & & & & & & & & & & 7 & 8 & 9 \\
\hline \multirow{3}{*}{$F_{3}$} & \multirow{3}{*}{1} & \multirow{3}{*}{2} & \multirow{3}{*}{3} & \multirow{3}{*}{2} & \multirow{3}{*}{3} & \multirow{3}{*}{1} & \multirow{3}{*}{3} & \multirow{3}{*}{1} & \multirow{3}{*}{2} & 1 & 2 & 3 \\
\hline & & & & & & & & & & 6 & 4 & 5 \\
\hline & & & & & & & & & & 8 & 9 & 7 \\
\hline \multirow{3}{*}{$F_{4}$} & \multirow{3}{*}{1} & \multirow{3}{*}{2} & \multirow{3}{*}{3} & \multirow{3}{*}{3} & \multirow{3}{*}{1} & \multirow{3}{*}{2} & \multirow{3}{*}{2} & \multirow{3}{*}{3} & & 1 & 2 & 3 \\
\hline & & & & & & & & & 1 & 5 & 6 & 4 \\
\hline & & & & & & & & & & 9 & 7 & 8 \\
\hline
\end{tabular}




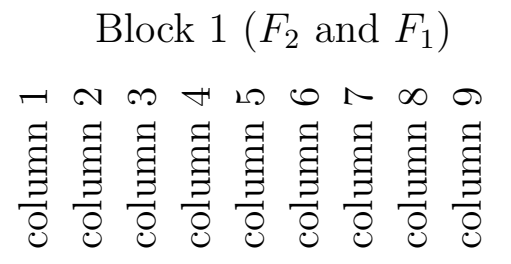

row $1 \quad 0 \quad 2131 \quad 0 \quad 22 \quad 32 \quad 0 \quad 2333$

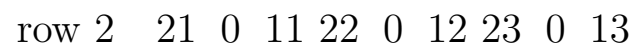

$\begin{array}{llllllllll}\text { row } 3 & 31 & 11 & 0 & 32 & 12 & 0 & 33 & 13 & 0\end{array}$

Block $3\left(F_{3}\right.$ and $\left.F_{2}\right)$

row $1 \quad 0 \quad 23 \quad 32 \quad 0 \quad 3312 \quad 0 \quad 1322$

row $2 \quad 23 \quad 0 \quad 1133 \quad 0 \quad 21 \quad 13 \quad 0 \quad 31$

row $3 \quad 3211 \quad 0 \quad 1221 \quad 0 \quad 22 \quad 31 \quad 0$
Block $2\left(F_{1}\right.$ and $\left.F_{4}\right)$

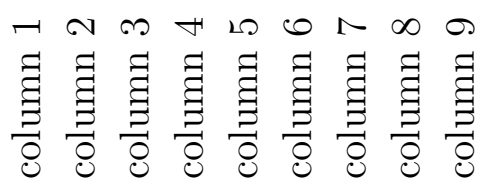

$\begin{array}{lllllllll}0 & 12 & 13 & 0 & 23 & 21 & 0 & 31 & 32\end{array}$

$\begin{array}{llllllllll}12 & 0 & 11 & 23 & 0 & 22 & 31 & 0 & 33\end{array}$

$1311 \quad 0 \quad 2122 \quad 0 \quad 32330$

Block $4\left(F_{4}\right.$ and $\left.F_{3}\right)$

$\begin{array}{lllllllll}0 & 22 & 33 & 0 & 31 & 12 & 0 & 13 & 21\end{array}$

$\begin{array}{llllllllll}22 & 0 & 11 & 31 & 0 & 23 & 13 & 0 & 32\end{array}$

$3311 \quad 0 \quad 1223 \quad 0 \quad 2132 \quad 0$

Figure 6: Design in Example 7

which specializes to $\mathrm{R}(t, t)$ when $h=2$. Now there is considerable flexibility in how we choose the pseudofactors for each block. Table 11 cannot show all the possibilities.

Example 8. Table 10 shows the canonical efficiency factors for four of the possible designs when $t=u=3$ and $n_{1}=2$. There is no information in stratum $B$. For each design, $A$ denotes the harmonic mean of the canonical efficiency factors in the bottom stratum, excluding the one for the contrast between the control and all other treatments.

The design in Table 10(a) uses Block 4 from Figure 6 together with a second block made from this by interchanging the roles of $F_{3}$ and $F_{4}$. This maximizes the efficiency for the main effects of $T$ and $U$ in the bottom stratum. The design in Table 10(b) uses Block 3 from Figure 6 together with a second block made by using $F_{4}$ and $F_{1}$ in place of $F_{3}$ and $F_{2}$. This gives A-optimality, among these designs, for treatment contrasts not involving the control. The design in Table 10(c) uses Blocks 3 and 4 from Figure 6. The design in Table 10(d) uses Block 3 from Figure 6 together with a second block made from this by using $F_{4}$ in place of $F_{3}$. 
Table 10: Canonical efficiency factors for four designs in Example 8

(a) (c)

\begin{tabular}{|c|c|c|c|c|c|c|c|c|}
\hline & \multicolumn{2}{|c|}{$\begin{array}{l}\rho(3)=\rho(4)=1 \\
\psi(3)=\psi(4)=1\end{array}$} & \multicolumn{2}{|c|}{$\begin{array}{l}\rho(1)=\rho(2)=1 \\
\psi(3)=\psi(4)=1\end{array}$} & \multicolumn{2}{|c|}{$\begin{array}{l}\rho(2)=\rho(3)=1 \\
\psi(3)=\psi(4)=1\end{array}$} & \multicolumn{2}{|c|}{$\begin{array}{c}\rho(2)=2 \\
\psi(3)=\psi(4)=1\end{array}$} \\
\hline & $T, U$ & $T \# U$ & $T, U$ & $T \# U$ & $T U F_{3}$ & $F_{4}$ & $T U$ & $T \# U$ \\
\hline$R[B]$ & 0 & $\frac{1}{12}$ & $\frac{1}{12}$ & 0 & $\begin{array}{lll}0 & \frac{1}{12} & \frac{1}{12}\end{array}$ & 0 & & 0 \\
\hline$C[B]$ & $\frac{1}{6}$ & $\frac{5}{12}$ & $\frac{1}{6}$ & $\frac{5}{12}$ & $\begin{array}{lll}\frac{1}{6} & \frac{1}{6} & \frac{5}{12}\end{array}$ & $\frac{5}{12}$ & & $\frac{5}{12}$ \\
\hline \multirow[t]{2}{*}{$R \# C[B]$} & $\frac{5}{6}$ & $\frac{1}{2}$ & $\frac{3}{4}$ & $\frac{7}{12}$ & $\begin{array}{lll}\frac{5}{6} & \frac{3}{4} & \frac{1}{2} \\
\end{array}$ & $\frac{7}{12}$ & & $\frac{7}{12}$ \\
\hline & \multicolumn{2}{|c|}{$A=0.625$} & \multicolumn{2}{|c|}{$A=0.656$} & \multicolumn{2}{|c|}{$A=0.640$} & \multicolumn{2}{|c|}{$A=0.653$} \\
\hline
\end{tabular}

\section{Discussion}

\subsection{Comparison with other designs in the literature}

The designs proposed in this paper give numerous possibilities for constructing near-factorial nested row-column designs for the assumed numbers of levels of two treatment factors. We propose designs whose treatments, apart from the control treatment, either have the structure typical for factorial experiments or have the trivial association scheme. The last possibility gives designs with supplemented balance, which have also been considered in other papers.

Gupta and Kageyama (1991) gave three constructions of designs with supplemented balance. A special case of the first one is our Construction 1 with $c=1$, which is just a Latin square for $v$ treatments. The general version of this construction uses $n_{1}$ blocks, each like Construction 1 but using only a subset of the non-control treatments, in such a way that the $n_{1}$ subsets form a balanced incomplete-block design. Typically $n_{1}$ is larger than the values in our constructions.

The second construction of Gupta and Kageyama (1991) uses $2 \times 2$ blocks, each with two replications of the control treatment on the diagonal. There is one block for each pair of non-control treatments, so again $n_{1}$ is large.

These two constructions both guarantee control orthogonality. Lacka and Kozłowska (2009) proposed other ways of constructing designs with supple- 


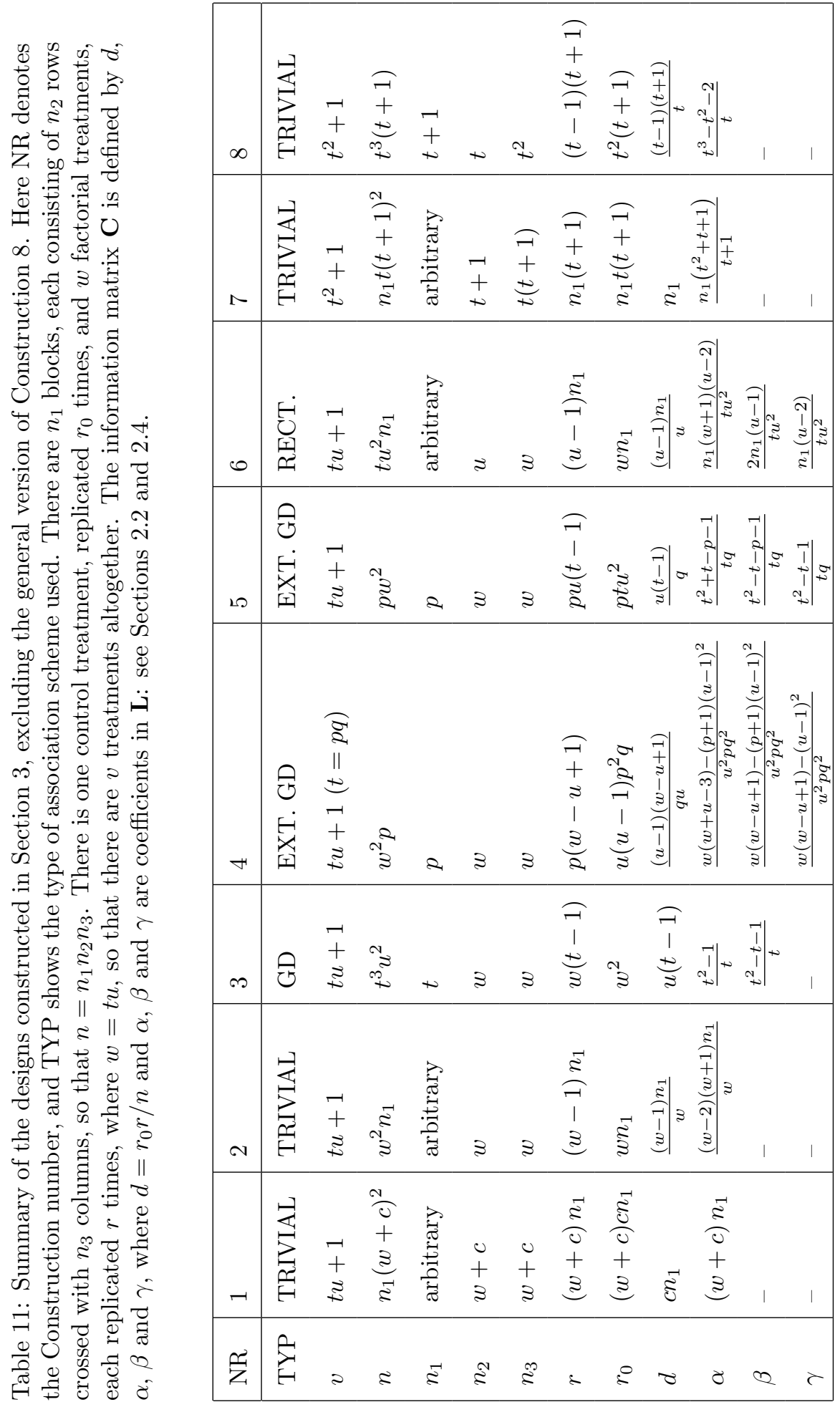


mented balance for blocks with two rows per block $\left(n_{2}=2\right)$, but without control orthogonality.

Supplemented group-divisible designs with nested rows and columns can be obtained by the method proposed by Kozłowska et al. (2011). Most of these designs do not have control orthogonality.

\subsection{Choosing a design for an experiment}

Of course, the values of the canonical efficiency factors are not the only parameters which should be considered when choosing the experimental design. Usually the values of $t, u, n_{1}, n_{2}$ and $n_{3}$ are specified, but different patterns of replication may give different variances for the contrasts of interest.

Example 9. Suppose that $t=2, u=3, n_{1}=2$ and $n_{2}=n_{3}=6$. Constructions 4 and 5 with $p=2$ and $q=1$ give two possible designs. In both, the contrast comparing the control treatment with all other treatments has full efficiency in the bottom stratum. In spite of this, the normalized variance for this contrast is smaller in the design made from Construction 5 $\left(0.0476 \sigma^{2}\right)$ than in the design made from Construction $4\left(0.0536 \sigma^{2}\right)$. For a contrast comparing the control with a single other treatment, the situation is the opposite: the normalized variance is $0.0892 \sigma^{2}$ if Construction 4 is used, or $0.1111 \sigma^{2}$ if Construction 5 is used. The contrasts for $U[T]$ have full efficiency in the bottom stratum in Construction 5, but not in Construction 4; however, Construction 4 gives a smaller normalized variance $\left(0.1364 \sigma^{2}\right)$ than Construction $5\left(0.1667 \sigma^{2}\right)$. See Table 12 .

Those two constructions differed in the pattern of treatment replication. However, this is not the only cause of different variances in different designs.

Example 10. Suppose that $t=u=n_{1}=2$ and $n_{2}=n_{3}=4$. Construction 2, and Construction 4 with $p=2$ and $q=1$, both give designs with $r_{0}=8$ and $r=6$. The only difference is the allocation of treatments to experimental units. Both designs have control orthogonality, and the normalized variance of the bottom-stratum estimator of the difference between the control and the average of the other treatments is $0.1333 \sigma^{2}$. However, for the contrast between the control and a single other treatment, the normalized variance is $0.1561 \sigma^{2}$ if Construction 4 is used, or $0.1583 \sigma^{2}$ if Construction 2 is used; the former has a smaller value of $\ell$. Similar comparisons are made for other types of contrast in Table 13. 
Table 12: Comparing designs with $t=2, u=3, n_{1}=2$ and $n_{2}=n_{3}=6$

\begin{tabular}{|c|c|c|c|c|c|c|c|c|}
\hline $\begin{array}{l}\text { Con- } \\
\text { struc- } \\
\text { tion }\end{array}$ & $r_{0}$ & $r$ & $\ell$ & $A$ & $\begin{array}{c}\text { varianc } \\
\text { contro } \\
\text { rest }\end{array}$ & $\begin{array}{l}\text { of norm } \\
\text { versus } \\
\text { another }\end{array}$ & lized cor & ast $/ \sigma^{2}$ \\
\hline 2 & 12 & 10 & 0.0893 & 0.9333 & 0.0857 & 0.0946 & 0.1071 & 0.1071 \\
\hline 4 & 24 & 8 & 0.1159 & 0.8987 & 0.0536 & 0.0892 & 0.1500 & 0.1364 \\
\hline 5 & 36 & 6 & 0.1667 & 0.8333 & 0.0476 & 0.1111 & 0.3333 & 0.1667 \\
\hline
\end{tabular}

Table 13: Comparing designs with $t=u=2, n_{1}=2$ and $n_{2}=n_{3}=4$

\begin{tabular}{|c|c|c|c|c|c|c|c|c|}
\hline $\begin{array}{l}\text { Con- } \\
\text { struc- } \\
\text { tion }\end{array}$ & $r_{0}$ & $r$ & $\ell$ & $A$ & $\begin{array}{c}\text { varianc } \\
\text { contro } \\
\text { rest }\end{array}$ & $\begin{array}{l}\text { of norm } \\
\text { versus } \\
\text { another }\end{array}$ & lized co & rast $/ \sigma^{2}$ \\
\hline 2 & 8 & 6 & 0.1500 & 0.8333 & 0.1333 & 0.1583 & 0.2000 & 0.2000 \\
\hline 4 & 8 & 6 & 0.1455 & 0.8594 & 0.1333 & 0.1561 & 0.1818 & 0.2000 \\
\hline 3 & 16 & 4 & 0.2500 & 0.7500 & 0.1000 & 0.1875 & 0.5000 & 0.2500 \\
\hline
\end{tabular}

The control treatment occurs $m_{3}$ times in each column. Since $r_{0}=$ $n_{1} n_{3} m_{3}$ and $r w=n_{1} n_{2} n_{3}-r_{0}$, we have $r_{0} / r=m_{3} w /\left(n_{2}-m_{3}\right)$. In an unblocked design, if it is only the contrasts between the control and individual other treatments that are important, we should try to make $r_{0} / r$ close to $\sqrt{w}$ (Hedayat et al., 1988). Since our designs have high canonical efficiency factors, this seems to be a good strategy here too, so we should try to make $\left(n_{2}-m_{3}\right) / m_{3}$ close to $\sqrt{w}$. If possible, we should try values of $m_{3}$ giving $\left(n_{2}-m_{3}\right) / m_{3}$ either side of $\sqrt{w}$. If $n_{2} \leq \sqrt{w}$, this suggests that designs with $m_{3}>1$ will not be good.

If contrasts with the control are less important, then $r_{0}$ (and hence $m_{3}$ ) should probably be chosen smaller than above.

Table 12 compares the two designs in Example 9, as well as a further design made from Construction 2. Those from Constructions 2 and 4 give $r_{0} / r$ close to, and either side of, $\sqrt{w}$, and the former is better for contrasts not involving the control.

Table 13 gives a similar comparison for the designs in Example 10 and a design made from Construction 3. The first two have $m_{3}=1$, so that 
Table 14: Comparing designs with $t=u=3, n_{1}=2, n_{2}=3$ and $n_{3}=9$

\begin{tabular}{|c|c|c|c|c|c|c|c|c|} 
& & & \multicolumn{5}{|c|}{ variance of normalized contrast $/ \sigma^{2}$} \\
Design & & & & & \multicolumn{4}{|c|}{ control versus } \\
rest & & & $\ell$ & $A$ & another & $T, U$ & $T \# U$ \\
\hline Table 10(b) & 18 & 4 & 0.3386 & 0.6562 & 0.0750 & 0.2110 & 0.3333 & 0.4286 \\
See text & 36 & 2 & 0.7778 & 0.5714 & 0.0750 & 0.4306 & 0.7500 & 1.0000
\end{tabular}

$\left(n_{2}-m_{3}\right) / m_{3}=3$, while the third has $m_{3}=2$ and $\left(n_{2}-m_{3}\right) / m_{3}=1$. These values are either side of 2 , which is $\sqrt{w}$, but the larger value of $m_{3}$ in the third design increases the variances of all contrasts except the one comparing the control with all the rest.

In Example 8, $n_{2}=3=\sqrt{w}$, and so it is unlikely that any design with $m_{3}>1$ will be competitive for any of the contrasts. Table 14 compares the design in Table 10(b) with another design which has $m_{3}=2$; the non-control treatments in the rows correspond to the levels of $F_{3}$ and $F_{4}$ in the two blocks. As expected, the second design performs poorly for all contrasts except the one comparing the control with the average of the rest.

There is no single rule for which construction should be chosen for an experiment. The decision should depend not only on the technical possibilities, but mainly on the goal of the experiment: for example, which contrasts are of most interest. This is especially true when several constructions are available for the chosen parameters. Depending on the purpose of the experiment, the experimenter will have a view about the relative importance of the various contrasts, and can use a weighted optimality criterion of the kind suggested by Morgan and Wang (2010); Wang and Morgan (2011) to choose the best design.

Acknowledgements. Both authors thank the editors and the referee for their helpful comments, suggestions and inspiration for further research. The authors also thank Queen Mary, University of London, the University of St Andrews and the Poznan University of Life Sciences for financial support. The second author was also supported by the British-Polish Young Scientists Programme, grant WAR/342/116. 


\section{References}

Bailey, R. A., 2004. Association Schemes: Designed experiments, algebra and combinatorics. Cambridge University Press, Cambridge.

Bailey, R. A., 2008. Design of Comparative Experiments. Cambridge University Press, Cambridge.

Bailey, R. A., Williams E. R., 2007. Optimal nested row-column designs with specified components. Biometrika 94, 459-468.

Ceranka, B., Mejza, S., 1979. On the efficiency factor for a contrast of treatment parameters. Biometrical Journal 21, 99-102.

Cochran, W. G., Cox, G. M., 1957. Experimental Designs (second edition). Wiley, New York.

Food and Agriculture Organization of the United Nations, 2006. International Code of Conduct on the Distribution and Use of Pesticides. Guidelines on Efficacy Evaluation for the Registration of Plant Protection Products. http://www.fao.org/fileadmin/templates/agphome/ documents/Pest_Pesticides/Code/Efficacy.pdf

Gupta, S., Kageyama, S., 1991. Type S designs with nested rows and columns. Metrika 38, 195-202.

Hedayat, A. S., Jacroux, M., Majumdar, D., 1988. Optimal designs for comparing test treatments with controls (with discussion). Statist. Sci. 3, 462491.

Houtman, A., Speed, T., 1983. Balance in designed experiments with orthogonal block structure. Ann. Statist. 11, 1069-1085.

Kozłowska, M., Łacka, A., Krawczyk, R., Kozłowski R. J., 2011. Some block designs with nested rows and columns for research on pesticide dose limitation. Environmetrics 22, 781-788.

Łacka, A., Kozłowska, M., 2009. Planning of factorial experiments in a block design with nested rows and columns for environmental research. Environmetrics 20, 730-742. 
Łacka, A., Kozłowska, M., Bogacka, B., 2009. Estimation and testing hypotheses in a block design with nested rows and columns. Biometrical Letters 46, 113-128.

Matthews, J. N. S., 2006. Introduction to Randomized Controlled Clinical Trials (second edition). Chapman \& Hall/CRC Press, Boca Raton.

Mejza, I., Mejza, S., 1994. Model building and analysis for block designs with nested rows and columns. Biometrical Journal 36, 327-340.

Mejza, S., 1992. On some aspects of general balance in designed experiments. Statistica 52, 263-278.

Morgan, J. P., Wang, X., 2010. Weighted optimality in designed experimentation. Journal of the American Statistical Association 105, 1566-1580.

Nelder, J. A., 1965a. The analysis of randomized experiments with orthogonal block structure. I. Block structure and the null analysis of variance. Proceedings of the Royal Society of London, Series A 283, 147-162.

Nelder, J. A., 1965b. The analysis of randomized experiments with orthogonal block structure. II. Treatment structure and the general analysis of variance. Proceedings of the Royal Society of London, Series A 283, 163178.

Nelder, J. A., 1968. The combination of information in generally balanced designs. Journal of the Royal Statistical Society, Series B 30, 303-311.

Pearce, S. C., 1960. Supplemented balance. Biometrika 47, 263-271.

Pearce, S. C., Caliński, T., Marshall, T. F. de C., 1974. The basic contrasts of an experimental design with special reference to the analysis of data. Biometrika 61, 449-460.

Shah, K. R., Sinha, B. K. (1989) Theory of Optimal Designs, Vol. 54 of Lecture Notes in Statistics. Springer, New York.

Wang, X., Morgan, J. P. (2011) Blocking, efficiency and weighted optimality. Biometrika 98, 967-978.

Yates, F. (1935) Complex experiments. Journal of the Royal Statistical Society, Supplement 2, 181-247. 